\title{
Serum lipid, lipoprotein and apolipoprotein changes in gestational diabetes mellitus: a cross-sectional and prospective study
}

\author{
E Koukkou, G F Watts, C Lowy
}

\begin{abstract}
Aims-To compare serum lipid, lipoprotein and apolipoprotein concentrations during and six to 12 months after pregnancy in control and diabetic women.

Methods-The serum lipid, lipoprotein and apolipoprotein concentrations were measured in 20 women with gestational diabetes mellitus (GDM) and 22 women with normal glucose tolerance (controls) during the third trimester of pregnancy and six to 12 months after delivery.

Results-During pregnancy the women with GDM had higher serum triglyceride (mean (95\% confidence interval (CI)), $2.91(2.22-3.51) v 2.1(1.75-2.52))$ but lower low density lipoprotein (LDL) cholesterol concentrations compared with controls (mean (SD), 3.08 (1.2) $v 4.01$ (1.1)). Total cholesterol, high density lipoprotein (HDL) cholesterol and apolipoprotein concentrations were not significantly different between the two groups. After pregnancy, total cholesterol, HDL cholesterol, triglyceride, and apolipoprotein $\mathrm{A1}$ and $\mathrm{B}$ decreased in a parallel manner, resulting in lower concentrations, comparable between the two groups. LDL cholesterol concentrations decreased after pregnancy in the controls (mean (SD), 4.01 (1.1) $v$ $2.69(0.6))$ but not in those with GDM (3.08 (1.2) $v 2.72(0.7))$. The change in lipid concentrations was not related to change in weight.
\end{abstract}

Conclusion-Development of diabetes during pregnancy induces a state of dyslipidaemia characterised by elevated triglyceride concentrations, as seen in other insulin resistance states. However, GDM seems to blunt the increase in LDL cholesterol during pregnancy and this requires further investigation. Whether the changes in lipoprotein metabolism in GDM are significant for the health status of the mother and the foetus requires further study.

(F Clin Pathol 1996;49:634-637)

Keywords: gestational diabetes, lipids, lipoproteins, apolipoproteins, follow up.

Change in lipoprotein metabolism during pregnancy is well established and is reflected by increased plasma concentrations of triglycerides, cholesterol and apolipoproteins. ${ }^{1-3}$ Diabetes mellitus is known to induce dyslipo- proteinaemia $^{45}$ and, therefore, it may be hypothesised that the emergence of diabetes? during pregnancy may further alter lipoprotein $\overrightarrow{0}$ metabolism. Although previous studies have shown that fasting hypertriglyceridaemia is accentuated and high density lipoprotein (HDL) cholesterol concentrations lower in diabetic pregnancies, ${ }^{6-8}$ other investigator ${ }_{\infty}^{\infty}$ were recently unable to demonstrate differ: ences in serum lipid, lipoprotein and apolipo $\rightarrow$ protein concentrations between normal and diabetic women. ${ }^{9}$ Moreover, there are still veryfew prospective studies and relatively little is known about the changes in lipid metabolisme during pregnancy complicated by gestationaff diabetes mellitus (GDM). ${ }^{10}$

In this study we examined the changes in lipid, lipoprotein and apolipoprotein concen-D trations during and six to 12 months after pregnancy in women with GDM and in controls with normal glucose tolerance.

\section{Methods}

Twenty women in the third trimester of pregnancy, who presented for an oral glucose tolerance test and in whom GDM waš diagnosed, were studied. The control group consisted of 22 women who also presented for screening for GDM and who had a normas glucose tolerance test. Both groups were stud₹ ied contemporaneously. Because of the knowng differences in lipid, lipoprotein and apolipo- $\rightarrow$ protein concentrations between different eth을 nic groups ${ }^{11}$ and because our patients werô racially heterogeneous (Caucasians, Asians and Africans/Afrocaribbeans), we matched the two groups for ethnicity. All subjects were on $\omega$ an ad libitum diet prior to testing. Fasting? venous blood was drawn with minimal stasio for measurement of serum total cholesterol ${ }_{\mathscr{Q}}^{\Phi}$ HDL cholesterol, triglycerides, and apolipo: proteins $A 1$ and $B$ (Apo $A 1$ and $B$, respectively), followed by the $75 \mathrm{~g}$ oral glucose toler ance test. Venous blood samples for plasmad glucose were drawn after 60 and 120 minutes Gestational diabetes was diagnosed according to the European Association for the Study of Diabetes (EASD) criteria, ${ }^{12}$ namely a plasmas glucose concentration $\geqslant 9 \mathrm{mmol} / 1120 \mathrm{~min} \frac{\bar{C}}{\overrightarrow{7}}$ utes after the $75 \mathrm{~g}$ oral glucose load. Maternal age, gestational age, parity, height, and weight were recorded on the day of the test. Body mass index $\left(B M I=\mathrm{kg} / \mathrm{m}^{2}\right)$ was derived for each subject. All women attended for a follow up visit 6 to 12 months after delivery and a fasting blood sample was drawn for 
Table 1 Clinical characteristics of women with GDM and with normal glucose tolerance (controls) during pregnancy. Results are expressed as mean (SD)

\begin{tabular}{lrrr}
\hline Clinical variables & $\begin{array}{l}G D M \\
(n=20)\end{array}$ & $\begin{array}{l}\text { Controls } \\
(n=22)\end{array}$ & $p$ value \\
\hline Age (years) & $30.5(4.4)$ & $29.9(5.1)$ & 0.7 \\
BMI (kg/m $\left.{ }^{2}\right)$ & $32.2(5.2)$ & $29.7(5.4)$ & 0.1 \\
Gestational age (weeks) & $31.3(3.4)$ & $30.0(3.4)$ & 0.24 \\
Parity (\%) & $3.0(1.4)$ & $3.4(2.2)$ & 0.48 \\
HbA1 (\%) & $6.6(0.7)$ & $6.0(0.7)$ & $<0.01$ \\
\hline
\end{tabular}

measurement of blood glucose, total cholesterol, HDL cholesterol, triglyceride, and Apo A1 and B concentrations. Total serum cholesterol and triglyceride concentrations were measured by enzymatic analysis on a DAX-96 analyser (Bayer Diagnostics, Basingstoke, UK): interassay coefficient of variation (CV) for cholesterol and triglycerides $<1.9 \%$ and $<2.8 \%$, respectively. HDL cholesterol was measured after dextran sulphate/ magnesium chloride precipitation of Apo B containing lipoproteins (Bayer Diagnostics): interassay $\mathrm{CV}<6.4 \%$. Apolipoproteins $\mathrm{Al}$ and $B$ were assayed by immunoturbidimetry using antiserum from Immuno (Sevenoaks, UK): interassay CVs $<2.5 \%$ and $<1.5 \%$, respectively. These analyses were performed on Cobas Fara or Cobas Bio analysers (Roche Diagnostic Systems, Welwyn Garden City, UK). LDL cholesterol was calculated by the Friedewald equation, excluding specimens with triglyceride concentrations higher than $4.5 \mathrm{mmol} / 1$ (three women with GDM and one control). ${ }^{13}$ The ratios of LDL:HDL cholesterol and LDL cholesterol:Apo B were calculated. The change in the lipid concentrations $(\delta)$ was calculated as the difference between the pregnant minus non-pregnant value. Triglyceride concentrations were log-transformed owing to their positively skewed distributions. Glycosylated haemoglobin (HbA1) was measured using an automatic high pressure liquid chromatography method (interassay CV $2.8 \%$, normal range 5.4-7.4\%).

Table 2 Serum lipid, lipoprotein and apolipoprotein concentrations in women with GDM and normal glucose tolerance (controls) during pregnancy. Results are expressed as mean $(S D)$

\begin{tabular}{lllc}
\hline & $G D M(n=20)$ & Controls $(n=22)$ & p value \\
\hline Total cholesterol $(\mathrm{mmol} / \mathrm{l})$ & $6.23(1.6)$ & $6.71(1.0)$ & 0.25 \\
HDL cholesterol $(\mathrm{mmol} / \mathrm{l})$ & $1.71(0.4)$ & $1.72(0.3)$ & 0.97 \\
LDL cholesterol $(\mathrm{mmol} / \mathrm{l})$ & $3.08(1.2)$ & $4.01(1.1)$ & $<0.01$ \\
Triglycerides $(\mathrm{mmol} / \mathrm{l})$ & $2.92(2.22-3.51)$ & $2.10(1.75-2.52)$ & $<0.05$ \\
Apolipoprotein A1 $(\mathrm{g} / \mathrm{l})$ & $1.93(0.2)$ & $1.88(0.3)$ & 0.61 \\
Apolipoprotein B (g/1) & $1.30(0.4)$ & $1.14(0.4)$ & 0.22 \\
LDL:HDL cholesterol & $1.77(0.6)$ & $2.35(0.8)$ & 0.017 \\
LDL cholesterol:Apo B & $0.94(0.25)$ & $1.19(0.22)$ & $<0.01$ \\
\hline
\end{tabular}

${ }^{\star}$ Geometric mean $(95 \% \mathrm{CI})$.

Table 3 Serum lipid, lipoprotein and apolipoprotein concentrations in women with GDM and normal glucose tolerance (controls) at follow up. Results are expressed as mean (SD)

\begin{tabular}{llll}
\hline & $G D M(n=20)$ & Controls $(n=22)$ & p value \\
\hline Total cholesterol $(\mathrm{mmol} / \mathrm{l})$ & $4.64(0.7)$ & $4.43(0.8)$ & 0.38 \\
HDL cholesterol $(\mathrm{mmol} / \mathrm{l})$ & $1.30(0.3)$ & $1.22(0.3)$ & 0.86 \\
LDL cholesterol $(\mathrm{mmol} / \mathrm{l})$ & $2.72(0.7)$ & $2.69(0.6)$ & 0.22 \\
Triglycerides ${ }^{\star}(\mathrm{mmol} / \mathrm{l})$ & $1.16(0.9-1.5)$ & $0.94(0.7-1.2)$ & 0.2 \\
Apolipoprotein A1 $(\mathrm{g} / \mathrm{l})$ & $1.07(0.3)$ & $0.94(0.1)$ & 0.09 \\
Apolipoprotein B (g/l) & $0.81(0.2)$ & $0.73(0.1)$ & 0.14 \\
LDL:HDL cholesterol & $2.23(1.0)$ & $2.36(0.8)$ & 0.67 \\
LDL cholesterol:Apo B & $1.30(0.16)$ & $1.46(0.16)$ & $<0.01$ \\
\hline
\end{tabular}

*Geometric mean (95\% CI).
STATISTICAL ANALYSIS

Comparisons were carried out using the paired and unpaired $t$ tests and values were expressed as mean (1 SD) or mean with $95 \%$ confidence intervals (CI) for log transformed data. Analysis of covariance was used to test the correlation between triglyceride concentrations and body weight and the correlation between lipid, lipoprotein and apolipoprotein concentrations during and after pregnancy.

\section{Results}

The two study groups were of similar age, gestational age, parity, and BMI but as expected the GDM group had higher fasting blood glucose and mean HBAl concentrations during pregnancy (table 1). The proportion of Caucasians, African/Afrocaribbeans and Asians in our groups was approximately 35, 40 and $25 \%$, respectively. When retested, all women had normal fasting blood glucose concentrations (mean (SD) GDM, 5.4 (0.3); controls, $5.2(0.3) \mathrm{mmol} / \mathrm{l})$.

\section{COMPARISON OF WOMEN WITH AND WITHOUT}

GDM DURING PREGNANCY

The serum lipid, lipoprotein and apolipoprotein concentrations and the ratios of these variables are given in table 2. Women with GDM had higher triglyceride, but lower LDL cholesterol concentrations compared with controls. No significant difference was found between the HDL cholesterol, and Apo A1 and $B$ concentrations in the two groups.

Total cholesterol concentrations were lower in the women with GDM; however, this difference did not reach significance. The LDL cholesterol:Apo B and LDL:HDL cholesterol ratios were significantly different between diabetic women and controls.

COMPARISON OF WOMEN WITH AND WITHOUT GDM SIX TO 12 MONTHS AFTER DELIVERY

Table 3 shows the lipid, lipoprotein and apolipoprotein concentrations and the ratios of these variables. No difference was found in the BMI (mean (SD) 28.7 (4.8) $v 27.6(4.5)$ ) and the lipid, lipoprotein and apolipoprotein concentrations between the women who previously had GDM and the women with normal glucose tolerance during pregnancy; triglyceride concentrations tended to be higher in the women who had had GDM. The LDL cholesterol:Apo B ratio remained significantly different between the two groups, whereas the LDL:HDL cholesterol ratio was similar.

COMPARISON OF THE LIPID PROFILES DURING AND AFTER PREGNANCY FOR EACH GROUP

There was a significant reduction in total cholesterol, HDL cholesterol, triglyceride, and Apo A1 and B concentrations after pregnancy in both groups (tables 2 and 3); the extent of the reduction for each variable was related to the pregnancy concentrations. The exception to this was LDL cholesterol concentrations in women with GDM, which were not significantly reduced after pregnancy (fig 1). 


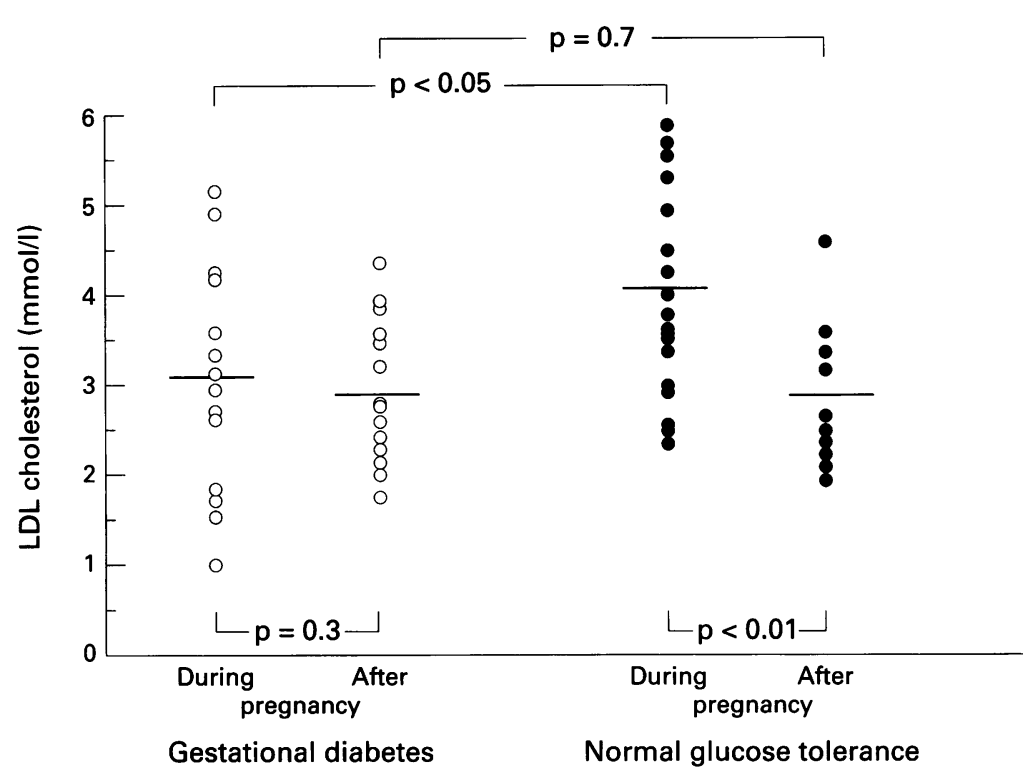

Figure 1 Change in plasma LDL cholesterol concentrations during and after pregnancy in women with GDM (O) and women with normal glucose tolerance ( $\bullet)$. Horizontal bars: mean values, $p$ values from paired/unpaired $t$ test.

\section{Discussion}

This prospective study shows that in pregnancy, women with GDM have significantly higher serum triglyceride but lower LDL cholesterol concentrations than women with normal glucose tolerance, after adjusting for age, BMI, parity, and ethnic group. There was also a significant difference in the LDL cholesterol:Apo B ratio between the two groups. After pregnancy the difference in serum lipid and lipoprotein concentrations between the two groups only persisted for the LDL cholesterol:Apo B ratio, which remained significantly lower in women who had had GDM.

Gestational diabetes has been shown to alter lipid metabolism. Knopp et $a l^{6}$ studied 22 women with GDM and 38 controls in the third trimester of pregnancy. They found increased plasma concentrations of triglycerides and very low density lipoprotein (VLDL) in the former, but no difference in plasma cholesterol concentrations. Their results are in agreement with those of Metzger et $a l^{8}$ and Hollinworth and Grundy, ${ }^{7}$ who also reported lower serum LDL cholesterol concentrations in women with GDM compared with controls. More recently, however, Montelogo et $a l^{9}$ did not find differences in serum lipid, lipoprotein or apolipoprotein concentrations between pregnant women with or without GDM. The discrepancies among studies may be the result of false negative tests of the null hypothesis in the studies with smaller sample sizes, as well as other methodological differences.

High serum oestrogen concentrations and increasing insulin resistance in late pregnancy are considered to be responsible for the hypertriglyceridaemia observed during "normal" pregnancy. In women with GDM increased insulin resistance may account for a further rise in triglyceride concentrations, as reported elsewhere. ${ }^{6-8}$ We cannot, however, fully explain the lower serum LDL cholesterol concentrations in women with GDM. Insulin resistance is associated with decreased LDL catabolism and a rise in plasma LDL cholesterol concentration, but the increased, direct removal of triglyceride enriched VLDL may lead to decreased production of LDL. ${ }^{4}$ The total pool $C$ of LDL may be further decreased by the effect $\cong$ of hyperoestrogenaemia on its catabolism. ${ }^{14}$ It has recently been pointed out ${ }^{15}$ that the Friedewald equation should not be used in non- 믐 insulin dependent diabetes mellitus, because there are compositional changes in VLDL that result in overestimation of LDL cholesterol. If $\bar{O}$ this also applies to GDM, we would expect that the women with GDM in the present study $\frac{\bar{\sigma}}{T}$ would have an even lower LDL cholesterol $\stackrel{\mathbb{\Omega}}{\Omega}$ concentration. Plasma Apo B concentrations क were comparable between women with and $\overrightarrow{0}$ without GDM, but the LDL cholesterol:Apo B ratio was lower in those with GDM, suggesting $\vec{\omega}$ a difference in particle composition, women with GDM having LDL which is cholesterol depleted and triglyceride enriched. Smaller $\overrightarrow{0}$ and denser LDL particles with decreased numbers of cholesterol ester molecules per $\stackrel{\omega}{A}$ particle have been reported in patients with 을 non-insulin dependent mellitus and impaired $\rightarrow$ glucose tolerance, ${ }^{16}$ as well as in women during the third trimester of pregnancy. ${ }^{17}$ The precise mechanism by which small, dense LDL arises $\stackrel{\oplus}{\rightarrow}$ is not known; there is evidence that LDL size $\overrightarrow{0}$ correlates negatively with plasma triglyceride content $^{1819}$ and plasma concentrations of insulin. ${ }^{16}$ After pregnancy, there was a trend for serum triglyceride concentrations to remain high and the LDL cholesterol:Apo B ratio remained significantly lower in the women who $\stackrel{\square}{\perp}$ had had GDM, indicating that the underlying $\overrightarrow{\vec{A}}$ cause of these abnormalities, although less prominent, persisted.

Our study has potential limitations. Firstly,

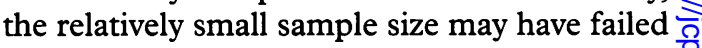
to detect significant differences in lipid concentrations after pregnancy. Secondly, our 3 inference that changes in lipoprotein concen- $\delta$ tration are the result of compositional changes $₹$ were not based on direct measurements of 욱 lipoprotein chemical composition. It would $\vec{D}$ have been more appropriate to measure the chemical composition or size of lipoproteins $N$ using ultracentrifugation or electrophoresis. However, we consider that our findings regard- $N$ ing LDL cholesterol concentrations are valid, $\omega$ given that measurement of LDL cholesterol concentrations by ultracentrifugation, in a dif- $\stackrel{\varrho}{C}$ ferent group of women with and without $\stackrel{\Phi}{\mathscr{Q}}$ GDM, yielded similar results (unpublished observations). We have also not provided ${ }_{0}^{\circ}$ evidence of the underlying mechanisms of $\underset{\mathbb{D}}{\mathbb{D}}$ abnormal lipoprotein metabolism and it would $\mathbb{\mathbb { D }}$ have been useful to measure oestrogen, insulin $\frac{\sigma}{\sigma}$ and free fatty acid concentrations. Stable isotope kinetic studies of lipid metabolism in GDM would be of considerable interest. Finally, we have not assessed dietary lipid intake, but as the patients in the present study received similar dietary advice we do not anticipate this to be a major confounding affect.

In conclusion, our findings indicate that GDM affects lipid and lipoprotein metabolism. The implications of our findings remain 
to be established. The smaller and more dense LDL particles are associated with increased risk of cardiovascular disease ${ }^{17}$ and are susceptible to oxidative modification which could in turn impair the physiology of nitric oxide $^{20}$ and potentially placental blood flow. Women with GDM are also at increased risk of developing frank diabetes later in life, which is known to eliminate or at least to attenuate the well known protection against ischaemic heart disease observed in women. ${ }^{21}$ Finally, although the lipid and lipoprotein changes are quite subtle and of short duration they may increase the risk of cardiovascular disease, especially with multiple pregnancies. ${ }^{22}$

The authors thank the staff of the Diabetic Day Care Centre for performing the glucose tolerance tests and the staff of the department of Chemical Pathology for performing the lipid analysis.

1 van Stiphout WAMJ, Hofman A, de Bruijn A. Serum lipids in young women before, during and after pregnancy. $A m \mathcal{F}$ Epidemiol 1987;126:922-8.

2 Jimenez DM, Pocovi M, Ramon-Cajal J, Romero MA, Martinez H, Grande F. Longitudinal study of plasma lipids and lipoprotein cholesterol in normal pregnancy and puerperium. Gynecol Obstet Invest 1988;25:158-64.

3 Mazurkiewicz J, Watts GF, Warburton FG, Slavin BM, Lowy C, Koukkou E. Serum lipids, lipoproteins and apolipoproteins in non-diabetic pregnancy. $\mathcal{F}$ Clin Pathol 1994; 47:728-31.

4 Howard BV. Lipoprotein metabolism in diabetes mellitus. $f$ Lipid Res 1987;28:613-28.

5 Orchard TJ. Dyslipoproteinemia and diabetes. Endocrinol Metab Clin North Am 1992;19:361-80.

6 Knopp RH, Chapman M, Bergelin R, Wahl PW, Warth MR, Irvine $\mathrm{S}$. Relationships of lipoprotein lipids to mild fasting hyperglycemia and diabetes in pregnancy. Diabetes Care 1980;3:416-20.

7 Hollinworth DR, Grundy SM. Pregnancy associated hypertriglyceridemia in normal and diabetic women. Diabetes 1982;31:1092-7.

8 Metzger BE, Phelps RL, Freinkel N, Navickas IA. Effects of gestational diabetes on diurnal profiles of plasma glucose, lipids and individual amino acids. Diabetes Care 1980; 3:402-9.
9 Montelogo A, Lasuncion MA, Pallardo LF, Herrera E. Longitudinal study of plasma lipoproteins and hormones during pregnancy in normal and diabetic women. Diabetes 1992;41:1651-9.

10 Kjos SL, Buchanan TA, Montoro M, Couslon A, Mestman $\mathrm{JH}$. Serum lipids within 36 months of delivery in women with recent gestational diabetes. Diabetes 1991;40:142-6.

11 Koukkou E, Watts GF, Mazurkiewicz J, Lowy C. Ethnic differences in lipid and lipoprotein metabolism in pregnan women of African and Caucasian origin. $\mathcal{F}$ Clin Patho 1994;47:1105-7.

12 National Diabetes data Group. Classification and diagnosis of diabetes mellitus and other categories of glucose intolerance. Diabetes 1979;28:1039-57.

13 Friedewald WT, Levy RI, Fredricson DS. Estimation of the concentration of low-density lipoprotein cholesterol in plasma without use of the preparative ultracentrifuge. Clin Chem 1972;18:499-502.

14 Sacks FM, Walsh BW. Sex hormones and lipoprotein metabolism. Current Opinion in Lipidology 1994;5:236-40.

15 Rubies-Prat J, Reverter JL, Senti M, Pedro-Botet J, Salinas $\mathrm{L}$, Lucas A, et al. Calculated low-density lipoprotein cholesterol should not be used for management of cholesterol should not be used for management of lipoprotein abnormalities in patien

16 Barakat HA, Carpender JW, Mclendon VD, Khazanie P, Legett N, Heath J, et al. Influence of obesity, impaired glucose tolerance and NIDDM on LDI structure and composition; possible link between hyperinsulinaemia and atherosclerosis. Diabetes 1990;39:1527-33.

17 Silliman K, Shore V, Forte TM. Hypertrigleridemia during late pregnancy is associated with the formation of smal dense low-density lipoproteins and the presence of large buoyant high-density lipoproteins. Metabolism 1994 43:1035-41.

18 Crouse JR, Parks JS, Schey HM, Kahl FR Studies of low density lipoprotein molecular weight in human beings with density lipoprotein molecular weight in human beings

19 McNamara JR, Campos J, Ordovas JM, Peterson J, Wilson PW, Schaefer EJ. Effect of gender, age and lipid status on low density lipoprotein subfraction distribution: results from the Framingham offspring study. Artherosclerosis 1987;7:489-90.

20 Flavahan NA. Atherosclerosis or lipoprotein-induced endothelial dysfunction. Potential mechanisms underlying reduction in EDRF/nitric oxide activity. Circulation reduction in ED

21 Barrett-Connor E, Wingard DL. Diabetes and heart disease in women. In: Eaker E, Packard B, Wenger N, eds. Coronary heart disease in women. New York: Haymarket Doyms, 1987:190-4.

22 Ness RB, Harris T, Cobb J, Flegal KM, Kelsey JL, Balanger $A, e t$ al. Number of pregnancies and the subsequent risk of cardiovascular disease. $N$ Engl f Med 1993;328:1528-33. 